\title{
Distributed Effects of Biological Sex Define Sex-Typical Motor Behavior in Caenorhabditis elegans
}

\author{
William R. Mowrey, ${ }^{1}$ Jessica R. Bennett, ${ }^{1}$ and Douglas S. Portman ${ }^{1,2,3}$ \\ ${ }^{1}$ Center for Neural Development and Disease, ${ }^{2}$ Department of Biomedical Genetics, and ${ }^{3}$ Department of Biology, University of Rochester, School of \\ Medicine and Dentistry, Rochester, New York 14642
}

Sex differences in shared behaviors (for example, locomotion and feeding) are a nearly universal feature of animal biology. Though these behaviors may share underlying neural programs, their kinematics can exhibit robust differences between males and females. The neural underpinnings of these differences are poorly understood because of the often-untested assumption that they are determined by sexspecific body morphology. Here, we address this issue in the nematode Caenorhabditis elegans, which features two sexes with distinct body morphologies but similar locomotor circuitry and body muscle. Quantitative behavioral analysis shows that $C$. elegans and related nematodes exhibit significant sex differences in the dynamics and geometry of locomotor body waves, such that the male is generally faster. Using a recently proposed model of locomotor wave propagation, we show that sex differences in both body mechanics and the intrinsic dynamics of the motor system can contribute to kinematic differences in distinct mechanical contexts. By genetically sexreversing the properties of specific tissues and cells, however, we find that sex-specific locomotor frequency in $C$. elegans is determined primarily by the functional modification of shared sensory neurons. Further, we find that sexual modification of body wall muscle together with the nervous system is required to alter body wave speed. Thus, rather than relying on a single focus of modification, sex differences in motor dynamics require independent modifications to multiple tissue types. Our results suggest shared motor behaviors may be sex-specifically optimized though distributed modifications to several aspects of morphology and physiology.

Key words: C. elegans; locomotion; motor control; sex differences

\section{Introduction}

Sex differences in behavior are a nearly universal feature of animal biology. As such, they provide outstanding examples of naturally occurring behavioral variation. Like the changes in behavior among closely related strains and species, sex differencesparticularly in shared behavioral programs - offer the opportunity to understand how evolution "tunes" complex systems to generate functional variation in behavior. While significant attention has been paid to the neural and anatomical substrates of behaviors that are sex-specific (e.g., courtship and copulation), relatively less is known about the mechanisms underlying sex differences in shared behaviors, such as feeding, locomotion, and

\footnotetext{
Received 0ct. 10, 2013; revised Nov. 5, 2013; accepted Nov. 7, 2013.

Author contributions: W.R.M. and D.S.P. designed research; W.R.M. and J.R.B. performed research; W.R.M., J.R.B., and D.S.P. analyzed data; W.R.M. and D.S.P. wrote the paper.

This work was supported by grants to D.S.P. from the National Science Foundation (IOS 0920024) and National Institutes of Health (NIH; R01 GM086456). W.R.M. was supported by National Institute of Mental Health Training Grant T32 MH065181 during part of this project. We are grateful to C. Cronin and P. Sternberg for worm tracking software and technical assistance, J. White and E. Jorgensen for sharing reagents, K. Meléndez-Cuadrado for help with assays of locomotor behavior, the Caenorhabditis Genetics Center, which is funded by the NIH Office of Research Infrastructure Programs (P40 0D010440), for providing nematode strains, and members of the Portman Laboratory for helpful comments on the manuscript.

The authors declare no competing financial interests.

Correspondence should be addressed to Douglas S. Portman at the above address. E-mail: douglas. portman@rochester.edu.

W. R. Mowrey's present address: Howard Hughes Medical Institute, Janelia Farm Research Campus, 19700 Helix Drive, Ashburn, VA 20147.

DOI:10.1523/JNEUROSCI.4352-13.2014

Copyright $\odot 2014$ the authors $\quad 0270-6474 / 14 / 341579-13 \$ 15.00 / 0$
}

aggression (Portman, 2007; Field and Whishaw, 2008; Anderson, 2012; Mowrey and Portman, 2012).

Whether sex-specific neural modifications play any significant role in shaping shared behaviors remains an outstanding question. Indeed, it is often assumed that differences in body morphology fully explain sexual variation observed in shared motor behaviors (Field and Whishaw, 2008). This assumption is supported by two general observations. First, the physiological properties of muscle and the mechanics of body-environment interaction shape the outcomes of neural motor commands in significant ways (Chiel and Beer, 1997; Alexander, 2003; Nishikawa et al., 2007). Second, neural circuits that support shared behavior often lack obvious anatomical specializations (Field and Whishaw, 2008; de Vries and Södersten, 2009). Emerging evidence, however, suggests that more subtle, functional modifications of neural circuitry may contribute to sex differences in shared behaviors (Mowrey and Portman, 2012). Work in Drosophila, for instance, has revealed that homologous motor circuitry in the two sexes is modified at either the level of fine-scale connectivity or cellular physiology to bring about sex differences in its dynamics (Clyne and Miesenböck, 2008). Such sex-specific modifications to the function of grossly similar circuits may be much more widespread than is realized, and could play a central role in establishing sex differences in shared behaviors. Rather than being an unintended consequence of morphological specialization, shared behaviors may instead be sex-specifically optimized through coordinated tuning of neural control, muscle 
properties, and body mechanics (Cohen, 1992; Chiel and Beer, 1997; Nishikawa et al., 2007).

Here, we address these issues by investigating the underpinnings of sex differences in the locomotor behavior of the nematode Caenorhabditis elegans. Locomotion in this species comprises the propagation of dorsoventral body waves along a viscoelastic, tube-like body. Locomotion in the smaller, more slender male has previously been described as "hyperactive" compared with the hermaphrodite (a modified female capable of transiently producing and storing self-sperm), though the mechanisms underlying this sex difference remain obscure (Hodgkin, 1974). Using a combination of biomechanical analysis and cellspecific genetic sex-reversal, we show that sex differences in C. elegans locomotor behavior are defined by coordinated modifications of shared sensory neurons and body wall muscle. Our results support the notion that the distributed modification of shared circuitry and musculature are integral to the sex-specific optimization of shared motor behavior.

\section{Materials and Methods}

Strains. Strains used in this study include the following: N2, CB3191, CB4856, CB4932, TR389, TR403, EM464, AF16, DR466 (him-5(e1490)), UR706 (fsEx271[Prab-3::fem-3(+)::SL2:::mCherry; Punc-122::GFP]), UR721 (fsEx317[Prab-3::tra-2 ${ }^{i c}::$ SL2:::mCherry; Punc-122::GFP]), UR708 (fsEx273 [Pmyo-3::fem-3(+)::SL2:::mCherry; Punc-122::GFP]), UR719 (fsEx315[Pmyo3::tra-2 ${ }^{i c}:: S L 2:: m$ Cherry; Psulp-3::GFP]), UR705 (fsEx270[Prab-3::fem-3 (+)::SL2:::mCherry; Pmyo-3::fem-3(+)::SL2:::mCherry; Punc-122::GFP]), UR717 (fsEx313[Prab-3::tra-2 ${ }^{i c}:: S L 2:: m$ Cherry; Pmyo-3::tra-2 ${ }^{i c}:: S L 2:: m$ Cherry; Punc-122::GFP]), UR822 (fsEx386[Prab-3::SL2::mCherry; Pmyo-3::SL2:: mCherry; Punc-122::GFP]), UR667 (him-5(e1490); fsEx301[Pacr-5::fem-3(+):: SL2:: mCherry; Punc-122::GFP]), UR667 (him-5(e1490); fsEx301[Pacr-5::fem-3 (+)::SL2:::mCherry; Punc-122::GFP]), UR668 (him-5(e1490); fsEx302[Pacr5::fem-3(+)::SL2:::mCherry; Punc-122::GFP]), UR510 (him-5(e1490); fsEx221[Punc-47::fem-3(+)::SL2:::mCherry; Punc-122::GFP]), UR511 (him5(e1490); fsEx222[Punc-47::fem-3(+)::SL2:::mCherry; Punc-122::GFP]), UR669 (him-5(e1490); fsEx303[Pnmr-1::fem-3(+)::SL2:::mCherry; Psra-11::fem-3 (+)::SL2:::mCherry; Punc-122::GFP]), UR670 (him-5(e1490); fsEx304[Pnmr1::fem-3(+)::SL2:::mCherry; Psra-11::fem-3(+)::SL2:::mCherry; Punc-122:: GFP]), UR226 (him-5(e1490); fsEx160[Posm-5::fem-3(+)::SL2:::mCherry; Punc-122::GFP]), UR227 (him-5(e1490); fsEx161[Posm-5::fem-3(+)::SL2::: mCherry; Punc-122::GFP]), UR247 (him-5(e1490); fsEx185[Posm-5::tra-2 ${ }^{i c}::$ SL2:: mCherry; Punc-122::GFP]), UR248 (him-5(e1490); fsEx186[Posm-5::tra$2^{i c}:: S L 2::$ mCherry; Punc-122:: GFP]), UR512 (him-5(e1490); fsEx223[Ptax4::fem-3(+)::SL2:::mCherry; Punc-122::GFP]), UR513 (him-5(e1490); fsEx224[Ptax-4::fem-3(+)::SL2::mCherry; Punc-122::GFP]), UR514 (him5(e1490); fsEx225[Posm-9::fem-3(+)::SL2:::mCherry; Punc-122::GFP]), UR515 (him-5(e1490); fsEx226[Posm-9::fem-3(+)::SL2:::mCherry; Punc-122::GFP]), UR470 (him-5(e1490); fsEx214[Pdat-1::fem-3(+)::SL2::mCherry; Punc122::GFP]), UR471 (him-5(e1490); fsEx215[Pdat-1::fem-3(+)::SL2:::mCherry; Punc-122::GFP]), UR466 (him-5(e1490); fsEx210[Ptph-1::fem-3(+)::SL2:: mCherry; Punc-122::GFP]), and UR467 (him-5(e1490); fsEx211[Ptph-1::fem-3 (+)::SL2:::mCherry; Punc-122::GFP]). Transgenic animals were generated using standard techniques (Mello and Fire, 1995), injecting $20 \mathrm{ng} / \mu \mathrm{l}$ of the sex-reversal construct of interest with $180 \mathrm{ng} / \mu \mathrm{l}$ of a coelomocyte-specific GFP reporter gene (Punc-122::GFP). For strains generated on the standard N2 background, males were obtained for experiments by mating. For neural subtype-specific strains, this was unnecessary as these strains were generated on a him-5(e1490) background, which generates males spontaneously at a high frequency $(\sim 30 \%)$ due to nondisjunction of the $\mathrm{X}$ chromosome. As partially feminized strains were often not competent to mate efficiently, nontransgenic males were mated to transgenic hermaphrodites to maintain males in these strains.

Molecular biology. All constructs generated for this study were made using the Multisite Gateway Cloning system (Invitrogen). To create 4-1R entry clones, we used PCR amplification of the promoters of the following genes: rab-3 (Nonet et al., 1997), myo-3 (Ardizzi and Epstein, 1987), osm-5 (Haycraft et al., 2001), acr-5 (Winnier et al., 1999), unc-47 (Eastman et al., 1999), nmr-1 (Brockie et al., 2001), sra-11 (Troemel et al.,
1995), dat-1 (Nass et al., 2002), tph-1 (Sze et al., 2000), osm-9 (Colbert et al., 1997), tax-4 (Komatsu et al., 1996). To generate expression clones, these constructs were recombined either with a bicistronic 1-2 entry clone coding for FEM-3 and mCherry (a gift from J. White and E. Jorgensen) and a 2-3 entry clone carrying the unc-54 3' UTR, or a 1-2 entry clone coding for a dominantly active fragment of the TRA-2 protein

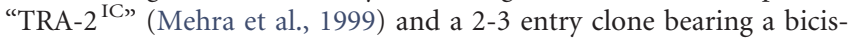
tronic mCherry gene and the unc-54 3' UTR.

Recording system. C. elegans locomotion was recorded using a semiautomated tracking system based on that described by Cronin et al. (2005), modified to capture images directly to hard disk rather than videocassette. Briefly, the system comprised a FireWire camera (Unibrain Fire-i 501b), microscope (Leica MZ7.5), computer (Dell Optiplex 640), and motorized stage (Ludl Electronic Products BioPoint2). The DigiTracker software developed by Cronin et al. (2005) was used to track worms during behavioral assays. The system was placed in a refrigerated incubator (Percival Scientific) to maintain consistent temperature during assays.

Assays of locomotor behavior. For the analysis of locomotor kinematics, behavior was recorded at a rate of 6 frames per second (fps) as solitary worms crawled for $1 \mathrm{~min}$ across the surface of a $90 \mathrm{~mm}$ plate of nematode growth medium (NGM) agar covered with a lawn of E. coli strain OP50. Worms were allowed $1 \mathrm{~min}$ to recover from the mechanical stimulus of being moved to the assay plate before the start of recording. All assays were performed at a temperature between 19.5 and $21^{\circ} \mathrm{C}$. Plates for assays were prepared by seeding with $1 \mathrm{ml}$ of OP50 grown to an $\mathrm{OD}_{600}$ of 1.0 , which was spread across the plate by agitation to create a continuous, featureless lawn. The lawn was allowed to grow $12-20 \mathrm{~h}$ at $20^{\circ} \mathrm{C}$ before the start of behavioral assays. Assays were carried out on young adult animals ( $4-8 \mathrm{~h}$ post-L4 stage) to avoid variations in behavior arising from strain differences in the gravidity of older hermaphrodites. For the developmental series, animals were synchronized by allowing 20 adult hermaphrodites to lay eggs on a culture plate for $1 \mathrm{~h}$. The eggs were then allowed to develop at $20^{\circ} \mathrm{C}$ for $40 \mathrm{~h}$ (L3 stage) or $50 \mathrm{~h}$ (L4 stage) before the start of the assay. Young adult animals were cultured as in other experiments. For transgenic strains, animals carrying the transgene were always tested in parallel with wild-type siblings. Neural subtype-specific strains were an exception, as these animals were tested in parallel with wild-type (i.e., DR466) controls from a laboratory stock.

Swimming experiments were carried out by placing worms in $200 \mu$ lof NGM buffer with $0.1 \%$ BSA that was sandwiched between two glass slides using no. 1 coverslips $(0.13-0.15 \mathrm{~mm}$ thickness) as spacers. BSA was included in the buffer to prevent worms from sticking to the glass slides while swimming. To modulate solution viscosity, we introduced high molecular weight dextran to the solution $(2,000,000$ molecular weight; Sigma-Aldrich) at 10,20 , or $30 \%(\mathrm{w} / \mathrm{v})$. The viscosity of these solutions was estimated based on values reported by Tirtaatmadja et al. (2001). Recordings were then carried out for $15 \mathrm{~s}$ at a rate of $25 \mathrm{fps}$. Environmental conditions were controlled as in standard locomotion assays.

Analysis of locomotor behavior. Recordings of solitary worm behavior were initially processed using the DigiRecognizer analysis package described by Cronin et al. (2005). Briefly, worms were recognized in collected images based on contrast, and reduced to a 13-point "spine" (an articulated line representing the posture of the worm). Further analysis was then carried out using custom software written in Matlab (Mathworks) implementing an analysis procedure similar to that described by Fang-Yen et al. (2010). Briefly, a cubic B-spline was fit to the 13-point spine, representing the posture of the worm in each frame as a 120-point two-dimensional curve. A set of worm-centered coordinates was determined for calculating kinematic metrics by calculating an "axis of propulsion" for each frame of movement. To do this, the mean slope of the 119 segments of the worm spine was calculated and then low-pass filtered to remove oscillations due to changes in worm shape over the wave cycle. Kinematic metrics were then calculated as follows: angle of attack $(\theta)$ was determined as the arctangent of the slope of each spine segment and curvature $(\kappa)$ was determined as the first derivative of the angle of attack along the arc length of the spine. Average frequency was calculated as the number of wave cycles over the period of recording, and average wave speed was taken as mean of linear fits to the zero-crossings of $\kappa$ in the 
body coordinate over time. Average wavelength $(\lambda / L)$ was calculated by dividing the wave speed by the frequency. Velocity was calculated by first calculating the velocity of each point of the spine using a central difference algorithm, and then projecting these velocity vectors onto the axis of propulsion. Averaging the velocity in the direction of propulsion along the spine yielded the mean velocity of the worm for each frame. Finally, the efficiency of propulsion was calculated as the ratio of velocity to wave speed. For metrics calculated at each time point, such as velocity, $\theta$, and $\kappa$, the behavior of individual worms was represented by the mode of their behavior rather than the mean. This was done to obviate the influence of rare, extreme values resulting from changes in the mode of locomotion, such as turning behaviors or reversal of direction. In rare instances, records that contained an excess of reversal behavior ( $\geq 5$ reversals) were censored, as this prevented accurate measurement of metrics describing forward locomotor behavior.

Measurement of body geometry. Body geometry was measured in terms of length and diameter for N2 animals at adulthood, L4, and L3, as well as for sex-reversed transgenic strains at adulthood (including transgenic and nontransgenic siblings). This was accomplished by placing animals in a drop of $50 \mathrm{~mm}$ muscimol (Sigma-Aldrich) on a $4 \%$ agarose pad on a glass slide. Animals were left $\sim 10 \mathrm{~min}$ in the drop to permit paralysis and relaxation of the body. The slides were then photographed (without coverslipping, to avoid compression and distortion of the body shape) on a Zeiss Axioskop microscope with differential interference contrast optics, using a $5 \times$ objective and a Hammamatsu ORCAII cooled CCD camera. Measurements of body size were made in Fiji (a distribution of ImageJ) by drawing a segmented line from the tip of the nose to the tail along the centerline of the worm. The tail was defined as the base of the tail spike in hermaphrodites and larvae, and as being just posterior to the cloaca in adult males. The width of the body was determined by drawing a line normal to the surface of the body midway from the head and tail (i.e., just anterior to the vulva in adult hermaphrodites).

Statistical analyses. Statistics were performed in Matlab. Comparisons on seven metrics were carried out for all assays of locomotor kinematics: velocity $(\mathrm{mm} / \mathrm{s})$, normalized wave speed $(L / \mathrm{s})$, frequency $(\mathrm{Hz})$, normalized wavelength $(\lambda / L)$, peak $\theta\left(^{\circ}\right)$, peak $\kappa\left(\mathrm{mm}^{-1}\right)$, and efficiency. In all cases simple two-sample $t$ tests were used to make planned comparisons between the groups, with the conservative Bonferroni correction for multiple comparisons used to adjust $\alpha$ (values reported in figure legends). For assays on the behavior of adult N2, wild isolates, and related nematodes, comparisons were only made between hermaphrodites and males within a group. Developmental analyses involved seven planned comparisons on each metric: comparing between adjacent stages within a sex and comparing between sexes within a stage. Assays on transgenic strains involved four comparisons on each metrics: comparing each line of transgenic animals to nontransgenic animals of the same sex, and comparing the sexes within transgenic or nontransgenic siblings. Swimming assays entailed four comparisons on each metric, as the sexes were compared at each level of viscosity.

Calculation of external and internal resistance to bending. As internal resistance to bending of the worm body is thought be determined primarily by the elastic cuticular shell that surrounds the worm, we estimated sex differences in this property by considering sex differences in the bending stiffness of the cuticle. In general, the bending stiffness of an elastic beam is given by $A=E I$, where $A$ is the bending stiffness, $E$ is the elastic modulus, and $I$ is the second moment of area of the beam. Assuming similar material properties of the cuticle in males and hermaphrodites, differences in the bending stiffness of the male and hermaphrodite body were estimated based on the ratio of the second moment of area of the cuticle $I$ in hermaphrodites and males. For a thin-walled cylinder like the worm's cuticular shell, $I=\pi r^{3} t$. Previous measurements on adult hermaphrodites suggest that cuticle thickness is proportional to the radius, yielding $I=\pi r^{4} / 44$ (Desai et al., 2003). Assuming this proportionality holds for males, sex differences in body geometry increase the second moment of area and bending stiffness significantly for the larger radius hermaphrodite body $\left(A_{\text {herm }}: A_{\text {male }}=2.4\right)$.

External resistance to bending of the worm body was estimated using the equations of resistive-force theory (Gray and Hancock, 1955). In resistive-force theory, external resistance to bending is given by the nor- mal drag coefficient $C_{N}=4 \pi \mu /[\ln (\lambda / r)+1 / 2]$, where $\mu$ is the viscosity of the medium, $\lambda$ is wavelength, and $r$ is body radius. This parameter scales forces along the short (or normal) axis of the body relative to the velocity of displacement along this axis $v_{N}$ as $F_{N}=C_{N} v_{N}$ (Gray and Hancock, 1955). The value of $C_{N}$ for animals swimming in saline buffer was calculated by direct measurement of the radius of the worm body and the wavelength of locomotion.

Levamisole sensitivity. To assay sensitivity to the acetylcholine receptor agonist levamisole, levamisole was dissolved in M9 buffer to a $50 \mu \mathrm{M}$ concentration. Aliquots of this solution were kept at $-20^{\circ} \mathrm{C}$ until the morning of the assay. The evening before the assay, L4 animals were picked to a fresh plate ( 20 worms per plate, separated by sex and genotype) and cultured at $20^{\circ} \mathrm{C}$ overnight. The next morning, 20 young adults were placed in a $30 \mu \mathrm{l}$ drop of levamisole on a glass slide. To limit evaporation, slides were kept in humid chamber (a lidded $150 \times 150 \mathrm{~mm}$ Petri dish) at room temperature $\left(20.5-22^{\circ} \mathrm{C}\right)$ during the assay. Animals were observed for $1 \mathrm{~min}$ periods every $15 \mathrm{~min}$ over a $90 \mathrm{~min}$ total assay period. Animals that failed to move during each observation period were scored as paralyzed; worms were not poked or prodded. Survival curves were generated using the Kaplan-Meier method and compared with the log-rank (Mantel-Cox) test using GraphPad Prism statistical software. $p$ values $<0.05$ were considered significant.

\section{Results}

\section{C. elegans exhibits robust, adult-specific sex differences in locomotor kinematics}

To better understand the mechanisms underlying sex differences in C. elegans locomotion, we first quantitated features of body morphology and locomotor kinematics in adult males and hermaphrodites. In this species, each sex exhibits characteristic body morphology at the young adult stage, with males being smaller and more slender than hermaphrodites (Fig. 1A). To extend previous qualitative descriptions of sex differences in adult $C$. elegans locomotion (Hodgkin, 1974), we used a modified version of the automated worm-tracking system developed by Cronin et al. (2005; see Materials and Methods). We found that young adult males and hermaphrodites crawling across the surface of a featureless culture plate exhibited distinct sex-typical characteristics in both speed and posture. Consistent with earlier observations (Hodgkin, 1974; Lipton et al., 2004), we found that locomotor velocity was faster in males compared with hermaphrodites (hermaphrodites: $0.15 \pm 0.0029 \mathrm{~mm} / \mathrm{s}$; males: $0.17 \pm 0.0025 \mathrm{~mm} / \mathrm{s}$, $t=-5.0, \mathrm{df}=142, p<0.001, n=72$ ), despite their smaller body size (see below). Additionally, male body-bend frequency was higher than that of hermaphrodites (hermaphrodites: $0.37 \pm$ $0.0052 \mathrm{~Hz}$; males: $0.45 \pm 0.0046 \mathrm{~Hz}, t=-11$, df $=142, p<$ 0.001 ; Fig. $1 C$ ). We also observed quantitative differences in the shape of body waves, given by the normalized wavelength $\lambda / L$ (the length of waves relative to body length; hermaphrodites: $0.56 \pm 0.0044$; males: $0.62 \pm 0.0046, t=-10, \mathrm{df}=142, p<$ 0.001 ), peak body curvature $\kappa$ (hermaphrodites: $8.6 \pm 0.10$ $\mathrm{mm}^{-1}$; males: $\left.11 \pm 0.11 \mathrm{~mm}^{-1}, t=-14, p<0.001\right)$, and peak angle of attack $\theta$ with respect to the axis of forward movement (hermaphrodites: $42 \pm 0.56^{\circ}$; males: $50 \pm 0.63^{\circ}, t=-9.8, \mathrm{df}=$ $142, p<0.001$; Fig. $1 B, C)$. Thus, although the anatomy of the $C$. elegans locomotor system is grossly sexually monomorphic, the sexes exhibit significant differences in specific features of locomotor behavior.

Resistive-force theory (Gray and Hancock, 1955; Lighthill, 1976) provides a framework for describing how sex differences in the kinematics of sinusoidal locomotion contribute to propulsion. C. elegans moves in a low Reynolds number regime (i.e., viscous forces dominate over inertial forces), meaning that reaction forces that propel the animal are proportional to the speed of its movement. Motion along the short and long axes of the body 
A

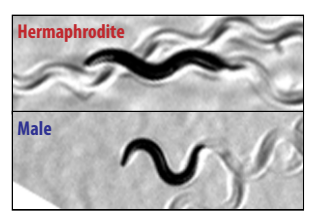

B

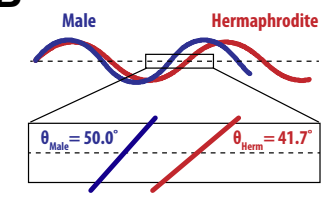

C
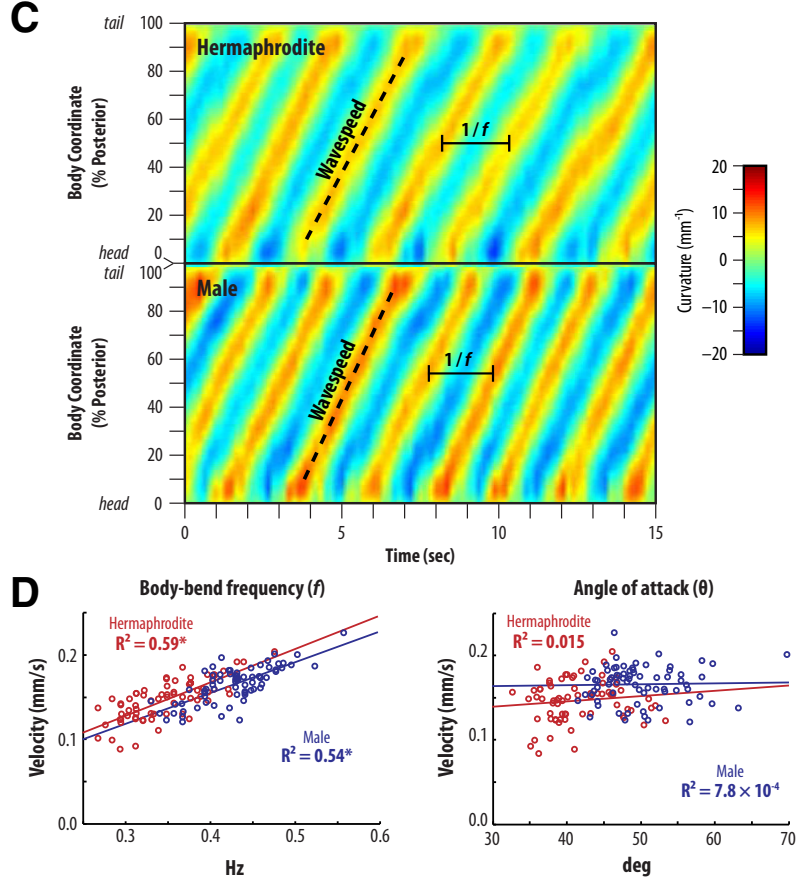

Figure 1. Sex differences in C. elegans locomotor behavior. A, Images of adult C. elegans crawling on a culture plate, illustrating characteristic sex differences in body morphology and waveform. $\boldsymbol{B}$, Sex differences in the locomotor waveform of young adults. Average waveforms show that the hermaphrodite (red) forms more waves along its body (indicative of a longer normalized wavelength) and exhibits lower curvature than the male (blue). Inset shows that waveform differences result in a higher peak angle of attack $(\theta)$ in males. $\boldsymbol{C}$, Color plots show waves of curvature propagating along the body of an individual hermaphrodite (top) and male (bottom) as a function of time and position. Males exhibit higher frequency of body waves, greater wave speed, and higher curvature. D, Scatter plots show the correlation of locomotor speed with wave frequency (left) and average angle of attack along the body (right). Speed is strongly correlated with frequency in both sexes (asterisk indicates $p<0.001$ for the slope term in linear regression), but correlates weakly or not at all with angle of attack ( $p=0.31$ and 0.82 for the slope in hermaphrodites and males, respectively).

are scaled by the normal and longitudinal drag coefficients $C_{N}$ and $C_{L}$, respectively. Resistive-force theory predicts that the efficiency of propulsion (defined as the ratio of worm velocity to wave speed) will increase with greater $C_{N} / C_{L}$ ratio, or with increasing angle of attack, according to the following equation (Gray and Hancock, 1955; Fang-Yen et al., 2010):

$$
\frac{V_{\text {worm }}}{V_{\text {wave }}}=\left(\frac{C_{N}}{C_{L}}-1\right)\left\langle\sin ^{2} \theta\right\rangle
$$

Although the average of $\sin ^{2} \theta$ along the body is significantly higher in males compared with hermaphrodites $(0.24 \pm 0.0039$ vs $0.17 \pm 0.0047, t=-12, p<0.001$ ), we find that the efficiency of propulsion is not significantly different between the sexes (hermaphrodites: $0.85 \pm 0.0085$; males: $0.85 \pm 0.0082, t=0.024$, $\mathrm{df}=142, p=0.98)$. This suggests a lower effective $C_{N} / C_{L}$ ratio for the male compared with the hermaphrodite, which may be a consequence of males' longer relative wavelength and smaller body radius (Gray and Hancock, 1955). Consistent with these observations, the increased speed of males appears to be driven largely by body-bend frequency rather than $\theta$, as the former exhibits a markedly stronger correlation with velocity across individuals than does the latter (Fig. 1D). Thus, it appears that the high-curvature male waveform functions primarily to maintain thrust with a lower effective $C_{N} / C_{L}$ ratio, while increased sinusoidal movement rate brings about an increased net velocity.

Before reaching reproductive maturity, C. elegans males and hermaphrodites pass through four larval stages. During this time, the overall body plan remains similar, while molting and new cuticle synthesis support body growth. Most sexually differentiated morphological characteristics begin to appear late in larval development, becoming fully elaborated at the final molt (Sulston and Horvitz, 1977; Emmons, 2005; Sternberg, 2005). Consistent with this, we found that sex differences in body-bend frequency and velocity were minimal in larvae and emerged at the larval-to-adult transition, coincident with the appearance of pronounced sex differences in body shape (Fig. 2A; data not shown). Notably, these differences appeared to emerge from a malespecific program, as hermaphrodite body-bend frequency and body-aspect ratio remained constant from L3 to adulthood (Fig. $2 A)$. In contrast, elevated male angle of attack appeared as early as L4 (Fig. 2A). Thus, we observed a strong developmental correlation between changes in body size and the modulation of locomotor behavior, raising the possibility that these properties could be mechanistically linked.

The adult-specificity of increased body-bend frequency and velocity suggests that these properties could be adaptations that support male reproductive fitness. Consistent with this possibility, we found similar sex differences in body-bend frequency and $\theta$ in nearly all of eight wild isolates we tested (Fig. 2B). Similar sex differences were also apparent in two other Caenorhabditis species, the androdioecious (male/hermaphrodite) C. briggsae as well as the gonochoristic (male/female) C. remanei (Fig. 2B). These results also indicate that the laboratory-derived polymorphism in the neuropeptide receptor gene $n p r-1$ in the C. elegans wild-type strain N2 (McGrath et al., 2009; Weber et al., 2010) is not the cause of sex differences in motor behavior.

\section{Sex differences in body wave geometry are determined by locomotor dynamics and body mechanics}

Recent work has shown that the propagation of $C$. elegans body waves is mediated, at least in part, by proprioceptive feedback through a specific motor neuron subtype (Wen et al., 2012). A simple model of proprioceptive coupling suggests that locomotor wavelength is determined by a small number of factors: the speed and spatial extent of coupling, the frequency of locomotion, and the ratio of external drag forces on the body to body stiffness. As noted above, $C$. elegans males differ from hermaphrodites in at least two of these factors, having higher locomotor frequency and a more slender body morphology. Thus, we asked whether these changes alone might be sufficient to explain sex differences in locomotor waveform.

Previous biomechanical analyses of $C$. elegans have indicated that body stiffness is primarily determined by the thin, elastic shell of cuticle that surrounds the body (Park et al., 2007; FangYen et al., 2010). Assuming cuticle composition and relative thickness to be approximately equivalent between the sexes, we found that the sex difference in body radius is predicted to create a 2.4-fold lower bending stiffness in the male (see Materials and Methods). In the proprioceptive coupling model, body stiffness is balanced by the drag forces acting across the normal axis of the body, characterized by the drag coefficient $C_{N}$. To estimate $C_{N}$, we examined the swimming of animals in dextran solutions of 
A
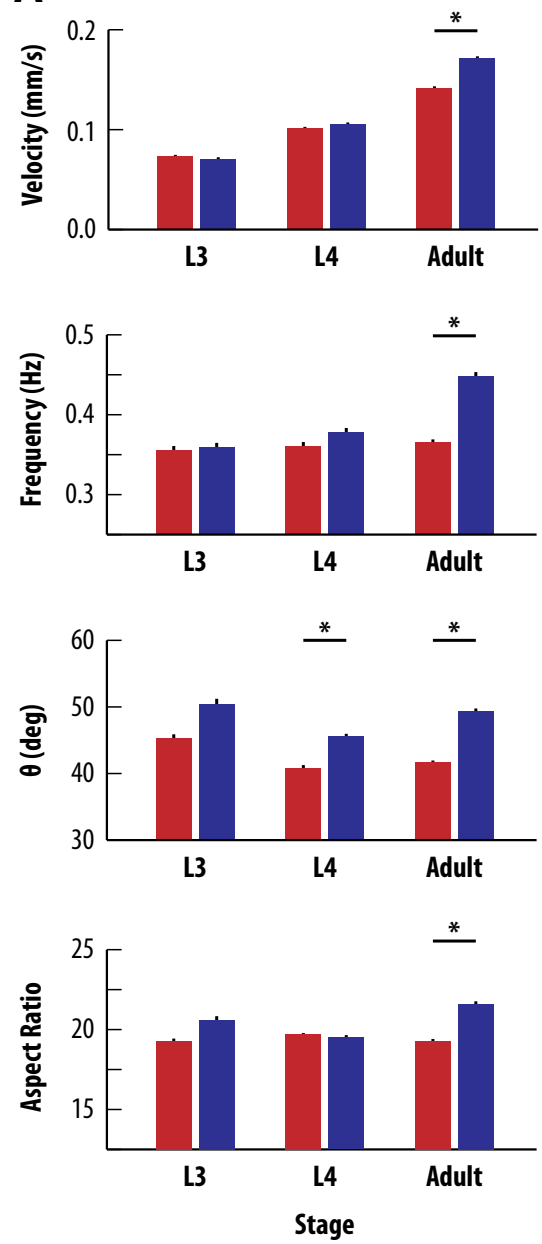

B
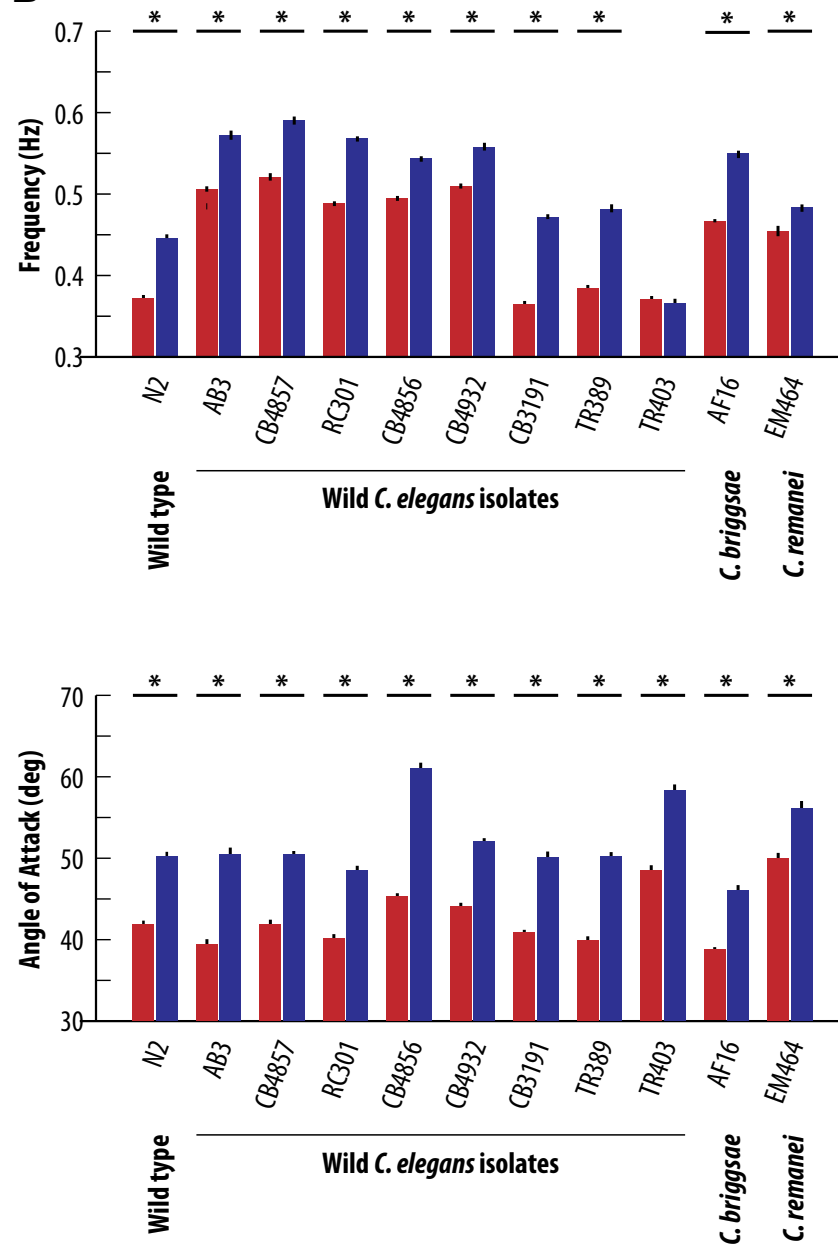

Hermaphrodite
Male

Figure 2. Sex differences in locomotion coincide with morphological differences over development and across species. $\boldsymbol{A}$, Sex differences in velocity, frequency, angle of attack, and slenderness all become most prominent at the adult stage. Sex differences emerge primarily through changes in male behavior and morphology during late larval development. Black bars indicate SEM. Asterisks indicate significant sex differences ( $p<0.0012$ for locomotor metrics, $p<0.0024$ for aspect ratio). $n=30$ for all groups in locomotion experiments. For groups in aspect ratio experiments, $n=$ 9 for L3 hermaphrodites, 11 for L3 males, 13 for L4 hermaphrodites, 11 for L4 males, 11 for adult hermaphrodites, and 9 for adult males. $\boldsymbol{B}$, Males exhibit increased locomotor frequency in nearly all wild isolates of $C$. elegans examined $\left({ }^{*} p<0.007\right)$. The sole exception is strain TR403, in which males exhibited uncoordinated locomotion. Higher male locomotor frequency was also observed in strain AF16 of the androdioecious (male/hermaphrodite) C. briggsae species, as well as in strain EM464 of the gonochoristic (male/female) nematode species C. remanei. Males exhibit higher peak angle of attack in all wild isolates of C. elegans examined, as well in isolates of C. briggsae and C. remanei ( $\left.{ }^{*} p<0.007\right) . n=24$ for all wild isolate C. elegans strains. $n=32$ for AF16 and EM464.

varying viscosities, following the approach of Fang-Yen et al. (2010) and Berri et al. (2009). Calculating this constant using the equations of resistive-force theory (Gray and Hancock, 1955; Lighthill, 1976), we found that $C_{N}$ differed by $<5 \%$ between males and hermaphrodites across viscosities varying by several orders of magnitude. Thus, we expect that under most conditions the influence of sex differences in body mechanics on locomotor wavelength will be dominated by differences in body stiffness.

Given that there is no evidence for sex differences in the gross anatomy of $C$. elegans ventral cord motor neurons, we assumed the spatial extent of coupling to be approximately consistent between sexes at a proportion of one-fifth of body length (Wen et al., 2012). Under this assumption, the time constant of coupling can be measured directly from the swimming of animals in saline buffer, where wave propagation is limited only by the worm's own neuromuscular and mechanical delays (Fang-Yen et al., 2010). In agreement with the observations of Wen et al. (2012), we found the time constant of wave propagation to be $75 \pm 1.1$ ms in hermaphrodites (mean \pm SEM). By contrast, faster male wave propagation yields a time constant of $53 \pm 2.2 \mathrm{~ms}$. This observation shows that a third factor-a sex difference in neuromuscular and/or mechanical properties that limit wave propagation speed-is likely to shape locomotor wavelength.

To determine whether the proprioceptive coupling model could be used to explain observed locomotor sex differences, we assessed the agreement of our kinematic data with the proprioceptive coupling model for animals swimming in solutions of varying viscosity. Locomotor wave speed and wavelength declined as viscosity increased in both sexes, though males retained higher values than hermaphrodites on these metrics under all conditions (Fig. $3 A, D$ ). Similarly, locomotor frequency also declined in both sexes with increasing viscosity, though sex differences in this metric were markedly reduced at the highest levels of external load (Fig. 3B). The proprioceptive coupling model predicted the overall trend of decreasing wavelength with viscosity, as well as the observed sex difference favoring longer wavelengths 
A

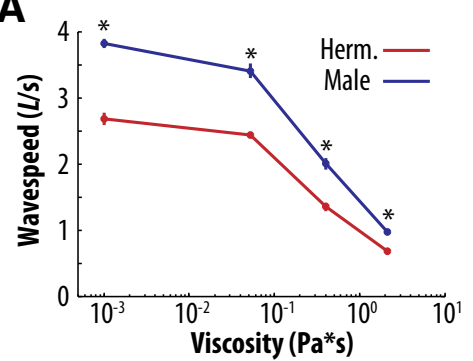

D

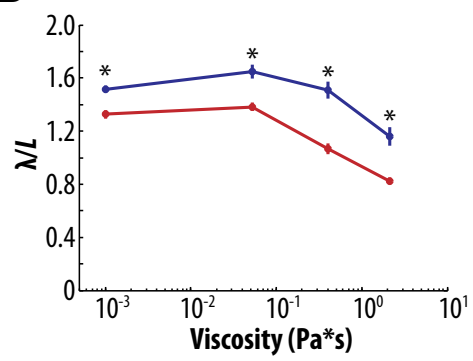

B

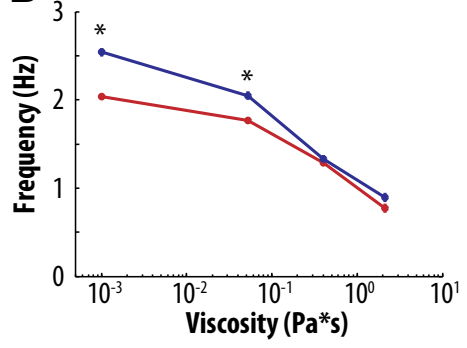

E

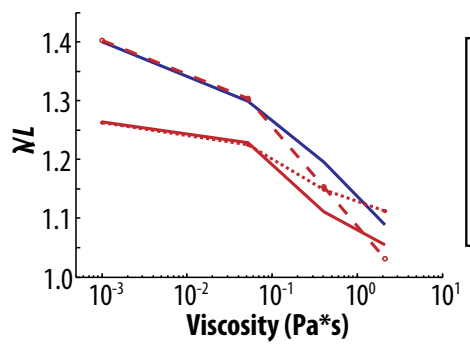

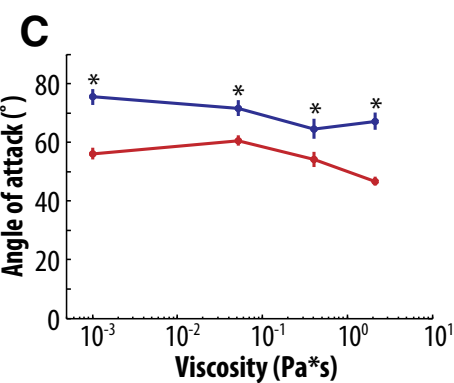

\begin{tabular}{|c|}
\hline Herm. model \\
Male model - \\
Herm. model \\
+ male mechanics \\
Herm. model \\
+ male dynamics
\end{tabular}

Figure 3. Sex differences in body mechanics and neuromuscular dynamics can both contribute to waveform disparities. $\boldsymbol{A}$, Wave speed sex differences are preserved across 10,000 -fold changes in mechanical load, despite significant decreases in wave speed with increasing viscosity. $\boldsymbol{B}$, Locomotor frequency sex differences are suppressed as locomotor frequency declines in both sexes at higher levels of mechanical load. $\boldsymbol{C}$, Angle of attack is conserved near the same sex-specific values across large changes in mechanical load. $\boldsymbol{D}$, Wavelength sex differences are maintained in all viscosities tested, though the absolute value of wavelength declines as viscosity increases. $\boldsymbol{E}$, Proprioceptive wave propagation model predicts the preservation of wavelength sex differences across viscosities. "Sex-reversal" of model parameters shows that sex differences at low mechanical load depend upon sex differences in neuromuscular delays in wave propagation, whereas at high loads sex differences are determined by body stiffness. Bars indicate SEM. Asterisks indicate significant sex differences $(p<0.0018)$. Different viscosity conditions were created by adding dextran to buffered saline solution at $0,10,20$, and $30 \%$ (w/v). $n=23$ for the $0 \%$ dextran treatment group, $n=14$ for $10 \%$ dextran, $n=15$ for $20 \%$ dextran, and $n=13$ for $30 \%$ dextran.

in males (Fig. 3E). Quantitative agreement of model predictions with the data were poor, however, as the model underestimated the observed wavelengths, particularly at higher viscosities (Fig. $3 E$ ). Nevertheless, the ability of the model to capture the essential disparity between male and hermaphrodite wavelengths suggests it can still serve as a useful tool for understanding how body mechanics, locomotor frequency, and the time constant of coupling contribute to this sex difference. "Sex-reversing" these parameters in the hermaphrodite model allowed us to assess how each contributes to sex differences in wavelength. We found that by sex-reversing the dynamics of behavior (that is, by substituting male estimates of the speed of coupling and frequency of wave propagation into the model of hermaphrodite wave propagation), sex differences were abolished at lower viscosities (Fig. 3E). At higher viscosities, however, marked sex differences in wavelength remained. By sex-reversing body mechanical variables (by substituting our estimates of male body stiffness and length into the model of hermaphrodite wave propagation), we observed the converse effect: sex differences at low viscosities remained, but were largely eliminated at high viscosities. These observations suggest a scenario in which the speed of wave propagation gives rise to waveform differences under low mechanical loads, but as load increases, body stiffness makes an increasing contribution to wavelength disparities.

An important aspect of locomotor kinematics that is not readily addressed using the proprioceptive coupling model is the angle of attack of the waveform. Unlike wavelength, the angle of attack depends on both locomotor frequency and the magnitude of muscle torques. Several authors have noted that the angle of attack appears to be conserved in nematode locomotion, both across different environments (Fang-Yen et al., 2010) and species (Gray and Lissmann, 1964). The mechanisms that regulate angle of attack remain incompletely understood, though proprioception by nonmotor neurons, such as the neuron DVA (Li et al.,
2006), is likely to play an important role. Significantly, we find that angle of attack is conserved in both males and hermaphrodites across viscosities, but at distinct levels (Fig. $3 C$ ). That male locomotion is characterized by higher angle of attack across 10,000 -fold variation in mechanical load strongly suggests this is a tightly regulated and functionally important property of male locomotor kinematics.

\section{Sexual modification of the nervous system determines locomotor frequency independently of body geometry} In principle, sex differences in C. elegans body mechanics, acting together with sexually isomorphic neural feedback mechanisms, could be sufficient to bring about sex-typical locomotor behavior. However, sexual modification of the motor system itself might also generate or optimize sex differences in the context of the distinct dynamics of male and hermaphrodite bodies. Such regulation of neuromuscular properties by biological sex could take several forms. Because the body-wall muscles that control sinusoidal locomotion bear no obvious sex differences in development or gross anatomy (White, 1988), sex differences in these cells would likely stem from modulation of ultrastructure or physiology. Likewise, most neural components of the locomotor system are shared by both sexes. While there are sex-specific motor neurons in the ventral nerve cord-VCs in hermaphrodites, CAs and CPs in males-these are thought to function primarily in the sex-specific behaviors of egg-laying and copulation, respectively, rather than locomotion (White et al., 1986; Loer and Kenyon, 1993; Barr and Garcia, 2006; Emmons, 2006; Schafer, 2006; Schindelman et al., 2006). Thus, neuromuscular sex differences with importance for general locomotion could arise via the functional modulation of shared circuits rather than through the contribution of sex-specific neuroanatomy.

To test this idea directly, we took advantage of the cellautonomous nature of C. elegans sexual differentiation (Wolff 
A
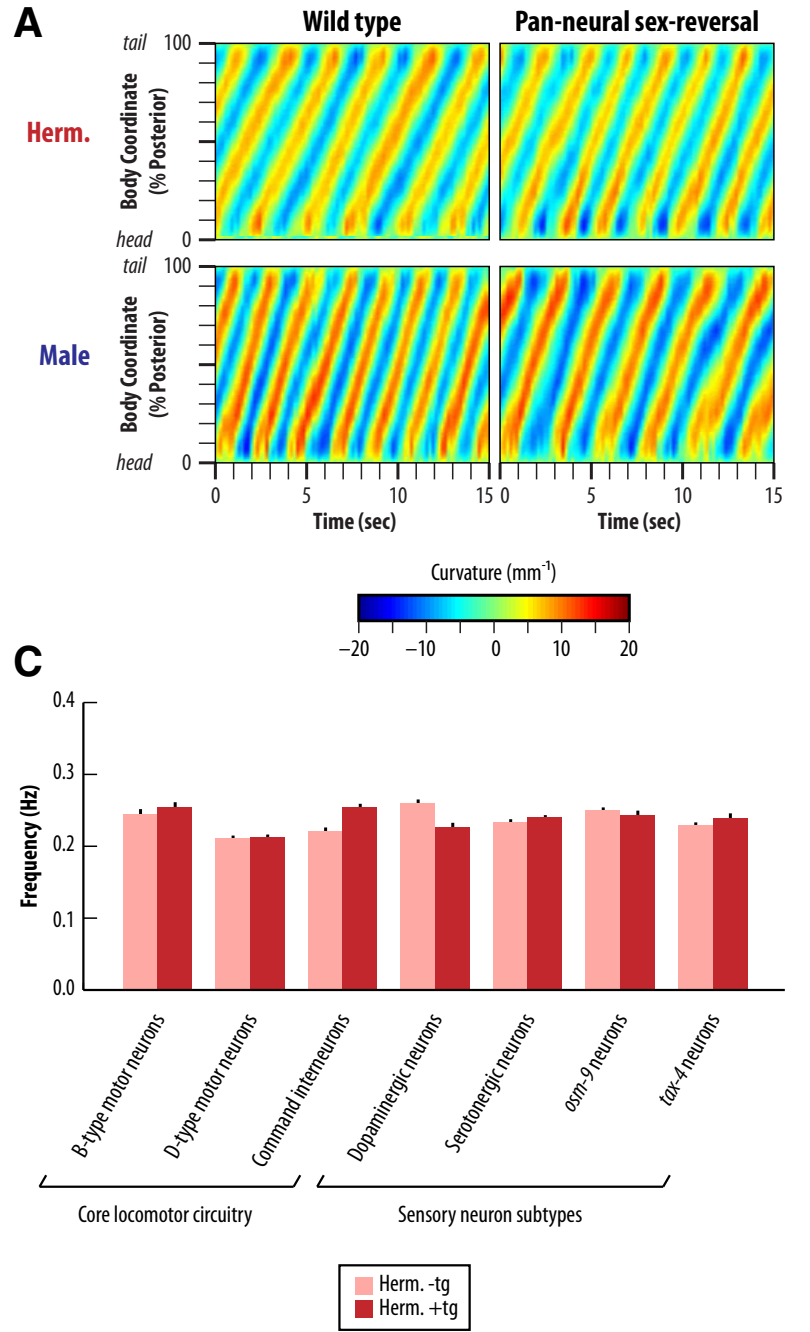

B

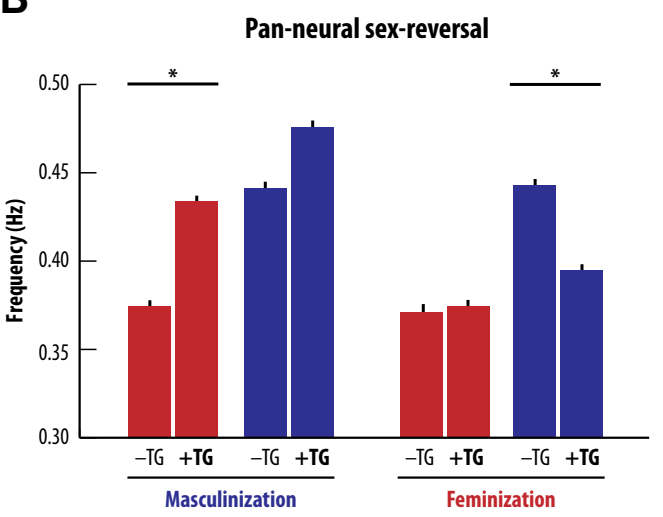

D

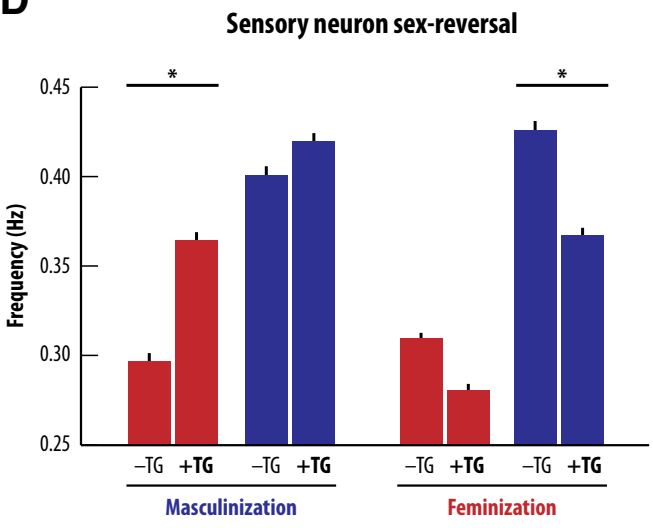

Figure 4. Locomotor frequency is regulated by sexual modification of the nervous system. $\boldsymbol{A}$, Color plots show propagating waves of curvature along the bodies of wild-type animals (left) or animals with sex-reversed nervous systems (right). Sex-reversal of the nervous system results in sex-reversed body-wave frequency. $\boldsymbol{B}$, Neural sex-reversal leads to opposite-sex locomotor frequency. For the neural masculinization groups: $-\mathrm{TG}$ (nontransgenic) hermaphrodites, $+\mathrm{TG}$ (transgenic) hermaphrodites, $-\mathrm{TG}$ males, and $+\mathrm{TG}$ males, $n=29,30,30$, and 29 , respectively. For the neural feminization groups: - TG hermaphrodites, + TG hermaphrodites, - TG males, and + TG males, $n=28$, 29, 28, and 26, respectively. C, Masculinization of core locomotor circuitry and specific subsets of sensory neurons fails to alter locomotor frequency. Graph depicts comparison between transgenic hermaphrodites carrying masculinization constructs (detailed in Results) and same-sex nontransgenic controls run in parallel. The number of animals in each group (nontransgenic and transgenic) were as follows: $n=18$ and 19 for B-type motor neurons, $n=16$ and 16 for GABAergic neurons, $n=16$ and 17 for command interneurons, $n=12$ and 12 for dopaminergic neurons, $n=14$ and 13 for serotonergic neurons, $n=11$ and 12 for osm- 9 neurons, and $n=16$ and 15 for tax-4 neurons. Two transgenic lines of each genotype were tested with similar results (only one line shown). $D$, Sex-reversal of ciliated sensory neurons leads to opposite-sex locomotor frequency. For the sensory neuron masculinization groups: - TG hermaphrodites, + TG hermaphrodites, $-\mathrm{TG}$ males, and $+\mathrm{TG}$ males, $n=13,18,20$, and 19 , respectively. For the sensory neuron feminization groups: - TG hermaphrodites, + TG hermaphrodites, - TG males, and + TG males, $n=18,19,22$, and 20, respectively. For $\boldsymbol{B}-\boldsymbol{D}$, bars represent mean \pm SEM. Color of bars indicates sex (red, hermaphrodite; blue, male). Asterisks indicate significant differences from same-sex sibling controls $(p<0.0018)$.

and Zarkower, 2008) to create sexually mosaic animals. Sex differences in C. elegans somatic tissues are controlled by the transcription factor TRA-1A, which acts cell-autonomously to repress male characteristics and promote hermaphrodite ones (Hodgkin, 1987; Hunter and Wood, 1990). Through its regulation by the sex-determination hierarchy, TRA-1A is highly active in XX hermaphrodites and weakly or inactive in X0 males (Wolff and Zarkower, 2008). To generate hermaphrodites in which specific tissues are genetically masculinized, we used cell-typespecific expression of the TRA-1A repressor FEM-3 as previously described (Lee and Portman, 2007; White et al., 2007). To feminize specific tissues in males, we expressed TRA-2 ${ }^{\text {IC }}$, a dominantly feminizing intracellular fragment of the TRA-1A activator TRA-2 (Lum et al., 2000). Importantly, because these sex-reversal transgenes are not activated until the onset of cell differentiation begins, they do not generally trigger the lineage alterations necessary to generate sex-specific cells of the opposite sex (Lee and Portman, 2007; White et al., 2007). Instead, this approach is expected to sex-reverse the functional properties of shared cells by activating or repressing targets of tra-1 in postmitotic cells.

We used tissue-specific sex-reversal to examine the role of sex-specific neural and muscle properties on the dynamics of locomotion. As shown in Figure $4 A$, neural sex-reversal had pronounced, reciprocal effects on locomotor dynamics, particularly on locomotor frequency. Quantification of this effect showed that hermaphrodites in which the entire nervous system was masculinized [Prab-3::fem-3(+)] exhibited significantly elevated locomotor frequency, similar to that of wild-type males (Fig. $4 A, B)$. Conversely, males in which the nervous system was feminized (Prab-3::tra- $2^{i c}$ ) exhibited a significantly reduced locomo- 
Table 1. Sex-reversal of neurons or muscle only weakly affects body morphology ${ }^{a}$

\begin{tabular}{|c|c|c|c|c|c|}
\hline Strain & Sex; genotype & $n$ & Length (mm) & Diameter (mm) & Aspect ratio \\
\hline \multirow[t]{4}{*}{ Neural masculinized } & Hermaphrodite; -TG & 10 & $946.1 \pm 15.1$ & $51.4 \pm 0.9$ & $18.4 \pm 0.1$ \\
\hline & Hermaphrodite; + TG & 11 & $940.9 \pm 15.1$ & $50.7 \pm 1.0$ & $18.6 \pm 0.4$ \\
\hline & Male; -TG & 11 & $809.9 \pm 10.0^{*}$ & $38.5 \pm 0.3^{*}$ & $21.0 \pm 0.2^{*}$ \\
\hline & Male; +TG & 10 & $799.1 \pm 14.9^{*}$ & $39.6 \pm 0.3^{*}$ & $21.2 \pm 0.4$ \\
\hline \multirow[t]{4}{*}{ Muscle masculinized } & Hermaphrodite; -TG & 11 & $931.4 \pm 12.0$ & $49.0 \pm 0.7$ & $19.0 \pm 0.2$ \\
\hline & Hermaphrodite; + TG & 11 & $923.0 \pm 11.5$ & $50.2 \pm 1.0$ & $18.4 \pm 0.2$ \\
\hline & Male; -TG & 10 & $795.2 \pm 8.1^{*}$ & $37.7 \pm 0.6^{*}$ & $21.1 \pm 0.3^{*}$ \\
\hline & Male; +TG & 9 & $769.9 \pm 16.1^{*}$ & $39.9 \pm 0.5^{*}$ & $19.3 \pm 0.5$ \\
\hline \multirow[t]{4}{*}{ Neural feminized } & Hermaphrodite; -TG & 10 & $966.3 \pm 10.5$ & $51.3 \pm 0.7$ & $18.1 \pm 0.1$ \\
\hline & Hermaphrodite; + TG & 8 & $900.1 \pm 20.0$ & $52.6 \pm 1.0$ & $17.1 \pm 0.5^{\dagger}$ \\
\hline & Male; -TG & 11 & $833.6 \pm 7.2^{*}$ & $39.2 \pm 0.5^{*}$ & $21.3 \pm 0.3^{*}$ \\
\hline & Male; +TG & 10 & $804.1 \pm 6.7^{*}$ & $42.8 \pm 0.6^{*}$ & $18.8 \pm 0.2^{* \dagger}$ \\
\hline \multirow[t]{4}{*}{ Muscle feminized } & Hermaphrodite; -TG & 11 & $911.0 \pm 11.6$ & $47.6 \pm 0.8$ & $19.2 \pm 0.2$ \\
\hline & Hermaphrodite; + TG & 10 & $910.3 \pm 21.2$ & $51.7 \pm 0.9^{\dagger}$ & $17.6 \pm 0.3^{\dagger}$ \\
\hline & Male; - TG & 9 & $817.9 \pm 4.0^{*}$ & $38.6 \pm 0.5^{*}$ & $21.2 \pm 0.3^{*}$ \\
\hline & Male; + TG & 10 & $832.3 \pm 7.8^{*}$ & $40.8 \pm 0.5^{*}$ & $20.4 \pm 0.2^{*}$ \\
\hline
\end{tabular}

${ }^{a}$ Transgenic (+TG) and nontransgenic ( $-\mathrm{TG}$ ) siblings of both sexes are compared on three measures of body geometry (mean \pm SEM) in strains carrying pan-neural or body wall muscle sex-reversal transgenes. Within-genotype effects of sex are indicated by asterisks ( $\left.{ }^{*} p<0.0042\right)$, and within-sex effects of the transgene are indicated by daggers.

Table 2. A control transgene lacking sex-reversal ORFs does not alter locomotor behavior ${ }^{a}$

\begin{tabular}{|c|c|c|c|c|c|c|c|}
\hline Sex; genotype & Velocity $(\mathrm{mm} / \mathrm{s})$ & Frequency (Hz) & $\lambda / L$ & Wave speed $(L / S)$ & $\theta\left(^{\circ}\right)$ & $\kappa\left(\mathrm{mm}^{-1}\right)$ & Efficiency \\
\hline Hermaphrodite; - TG $(n=28)$ & $0.15 \pm 0.0099$ & $0.38 \pm 0.011$ & $0.55 \pm 0.0076$ & $0.21 \pm 0.0070$ & $42 \pm 0.79$ & $8.8 \pm 0.18$ & $0.86 \pm 0.017$ \\
\hline Hermaphrodite; + TG $(n=30)$ & $0.15 \pm 0.015$ & $0.39 \pm 0.0082$ & $0.56 \pm 0.0063$ & $0.21 \pm 0.0052$ & $40 \pm 0.65$ & $8.4 \pm 0.13$ & $0.85 \pm 0.018$ \\
\hline Male; - TG $(n=26)$ & $0.19 \pm 0.016^{*}$ & $0.50 \pm 0.0094^{*}$ & $0.63 \pm 0.0048^{*}$ & $0.31 \pm 0.0070^{*}$ & $49 \pm 0.89^{*}$ & $11 \pm 0.14^{*}$ & $0.84 \pm 0.0086$ \\
\hline Male; + TG $(n=30)$ & $0.19 \pm 0.014^{*}$ & $0.51 \pm 0.0078^{*}$ & $0.64 \pm 0.056^{*}$ & $0.33 \pm 0.0059^{*}$ & $48 \pm 0.79^{*}$ & $11 \pm 0.15^{*}$ & $0.85 \pm 0.0061$ \\
\hline
\end{tabular}

${ }^{a}$ Transgenic (+TG) and nontransgenic (-TG) siblings of both sexes are compared on six locomotor metrics (mean + SEM) for strain UR822 ( fsEx386 [Prab-3::SL2::mCherry; Pmy0-3::SL2::m Cherry; Punc-122::GFP]), which carries a contro transgene for pan-neural and body wall muscle sex-reversal where the sex-reversal ORFs are absent. While within-genotype sex differences between males and hermaphrodites were readily detected on multiple measures ( $\left.{ }^{*} p<0.0018\right)$, no significant within-sex effects of the transgene were found.

tor frequency, only slightly higher than that of wild-type hermaphrodites (Fig. 4A,B). Importantly, these changes in behavior were not accompanied by appreciable sex-reversal of body geometry (Table 1). By contrast, control transgenic lines carrying constructs lacking the open reading frame encoding FEM-3 or TRA-2 ${ }^{\text {IC }}$ showed no change in locomotor frequency, confirming that the observed effects were strictly a consequence of sexreversal (Table 2). Together, these reciprocal effects indicate that the sex-specific modification of shared neural circuitry is largely sufficient to give rise to sex differences in body-bend frequency.

To better understand the neural mechanisms that determine sex-typical body-bend rates, we used subtype-specific promoters to sex-reverse subpopulations of neurons. We first focused on the core locomotor circuitry of the worm, comprising both cholinergic (A and B types) and GABAergic (D type) motor neurons, as well as command interneurons that control whether the forward or reverse motor circuit is active (Chalfie et al., 1985). In contrast to masculinization of the entire nervous system, masculinizing components of this circuitry critical for forward locomotion, including B-type motor neurons [Pacr-5::fem-3(+)], D-type motor neurons [Punc-47::fem-3(+)], and forward command interneurons [Pnmr-1::fem-3(+); Psra-11::fem-3(+)], had no detectable effect on locomotor frequency (Fig. 4C). Notably, the activity of the motor circuit is also influenced by a number of sensory inputs. To broadly survey whether sexual modification of these inputs could play a role in regulating locomotor frequency, we sex-reversed all ciliated sensory neurons (Perkins et al., 1986), most of which have chemosensory and mechanosensory functions. Surprisingly, this manipulation elicited strong, reciprocal effects on body-bend frequency: sensory-masculinized hermaphrodites had a male-like frequency, and sensory-feminized males slowed to a rate similar to that of wild-type hermaphrodites (Fig. $4 D)$. Several sensory neuron subtypes have been previously im- plicated in the regulation of locomotor frequency, including dopaminergic and serotonergic sensory neurons (Sawin et al., 2000). However, targeted masculinization of these sensory neurons subtypes [Pdat-1::fem-3(+) and Ptph-1::fem-3(+)], as well as broad subsets of gustatory and olfactory neurons [Ptax-4::fem-3(+) and Posm-9::fem-3(+)], failed to alter locomotor frequency. Thus, while the specific sensory neurons targeted by sexual modulation are unknown, the sex-specific regulation of sensory function is a key source of the sex-typical body-bend frequency of adult $C$. elegans males and hermaphrodites.

\section{Sexual modification of body wall muscle regulates the dynamics of wave propagation}

Although neural sex-reversal had strong effects on locomotor frequency, it did not generate waveforms typical of the opposite sex. Rather, hermaphrodites with a masculinized nervous system exhibited a pronounced decrease in wavelength, while neural sex-reversed males showed longer body waves (Fig. $5 A$ ). Further, angle of attack was altered in these animals in a manner that was also inconsistent with a sex-reversal phenotype (hermaphrodite masc. tg. $(-): 41 \pm 0.90$; hermaphrodite ${ }_{\text {masc. tg. }(+)}$ : $37 \pm 0.50, t=3.8, \mathrm{df}=57, p<0.001 ;$ male $_{\text {fem. tg. }(-)}: 50 \pm$ 0.90, male $\left._{\text {fem. tg. }(+)}: 48 \pm 0.93, t=1.3, \mathrm{df}=52, p=0.19\right)$. As the body geometry of neural sex-reversed animals was not substantially different from that of nontransgenic siblings, this phenotype is unlikely to result from altered body mechanics. As above, we observed no change in the behavior of animals carrying control constructs lacking sex-reversal coding sequence (Table 2), demonstrating that these seemingly paradoxical waveform changes are a specific consequence of neural sex-reversal.

An interesting possibility is that these changes may be a consequence of altered locomotor frequency itself. As discussed above, recent work has shown that body-wave propagation is 
A
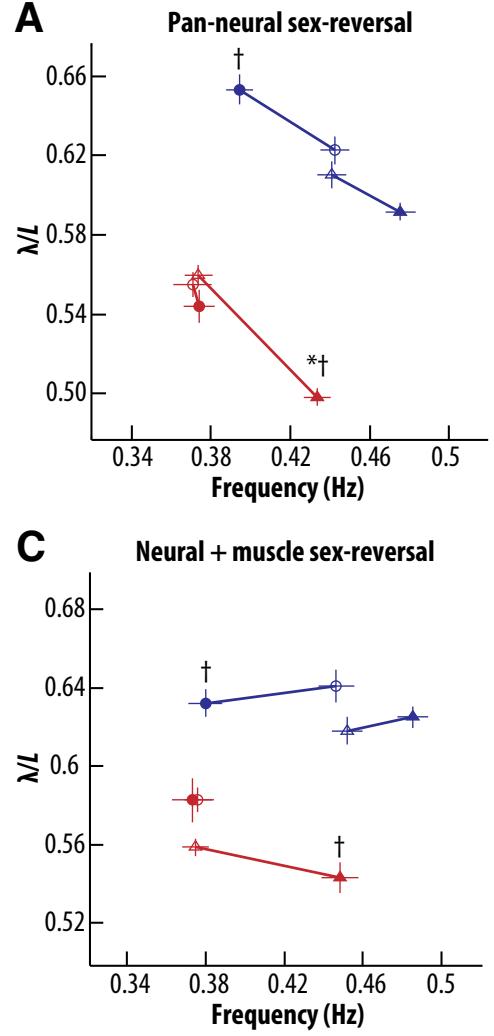

B

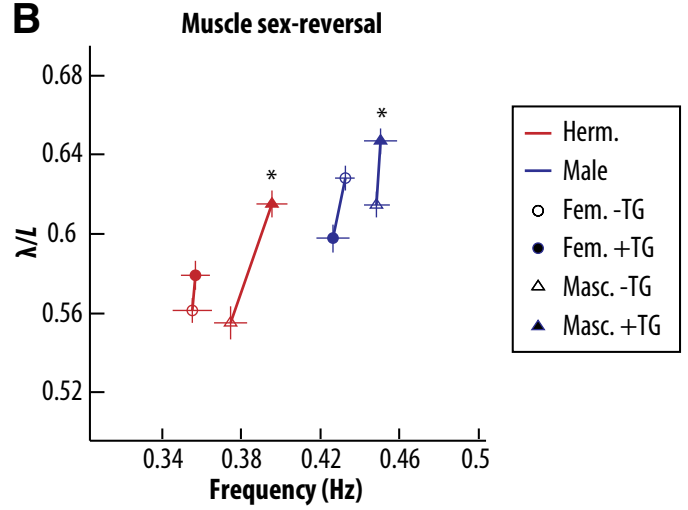

D

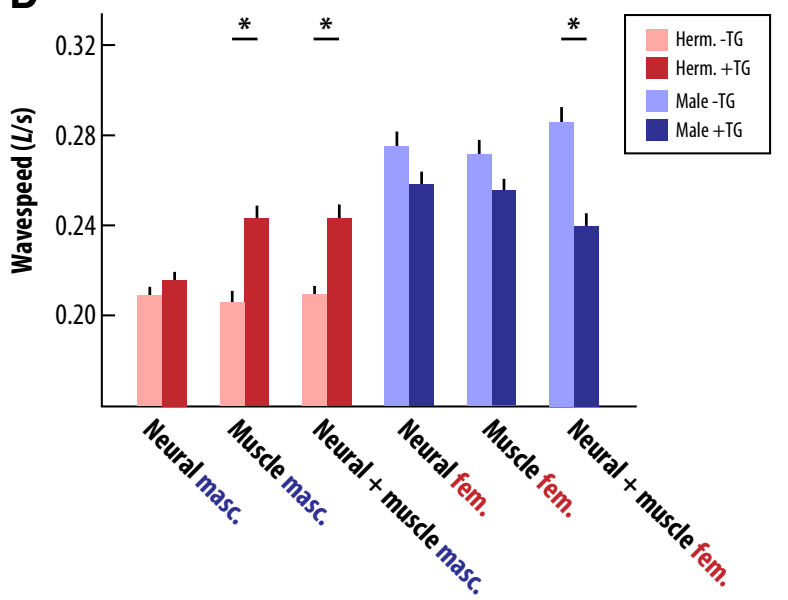

Figure 5. Sexual modification of muscle alters the dynamics of wave propagation. $A$, Pan-neural sex-reversal induces shifts in wavelength that are not consistent with a sex-reversal phenotype, but are proportional to changes in locomotor frequency. For numbers of animals tested, see legend for Figure 4. $\boldsymbol{B}$, Sex-reversal of body wall muscle in either sex induces reciprocal changes in wavelength, accompanied by slight or no changes in locomotor frequency. For the muscle-masculinization groups: - TG (nontransgenic) hermaphrodites, $+\mathrm{TG}$ (transgenic) hermaphrodites, $-\mathrm{TG}$ males, and $+\mathrm{TG}$ males, $n=30,29,28$, and 28, respectively. For the muscle-feminization groups: $-\mathrm{TG}$ hermaphrodites, $+\mathrm{TG}$ hermaphrodites, $-\mathrm{TG}$ males, and $+\mathrm{TG}$ males, $n=27,29,28$, and 28 , respectively. C, Simultaneous sex-reversal of neurons and muscle results in sex-reversed locomotor frequency, while preserving sex-typical wavelength. For the neural-masculinization and muscle-masculinization groups: - TG hermaphrodites, +TG hermaphrodites, - TG males, and +TG males, $n=30,29,30$, and 29, respectively. For the neural-feminization and musclefeminization groups: $-\mathrm{TG}$ hermaphrodites, $+\mathrm{TG}$ hermaphrodites, $-\mathrm{TG}$ males, and $+\mathrm{TG}$ males, $n=24,25,25$, and 23 , respectively. For $\boldsymbol{A}-\boldsymbol{C}$, markers indicate mean \pm SEM. Asterisks indicate significant within-sex differences in wavelength. Daggers represent within-sex differences in frequency ( $p<0.0018$, Bonferroni corrected $p$ value). $\boldsymbol{D}$, Sex-reversal of body wall muscle together with neurons induces changes in wave speed, whereas sex-reversal of the nervous system alone fails to do so. Bars represent mean $\pm S E M$. Asterisks indicate significant differences from same sex sibling controls ( $p<0.0018$, Bonferroni corrected $p$ value).

mediated by proprioceptive coupling between body segments (Wen et al., 2012). A simple linear model of this coupling suggests that locomotor wavelength should be a monotonically decreasing function of frequency (Wen et al., 2012). By plotting the relationship between locomotor wavelength and frequency, we observed that the increased locomotor frequency caused by masculinization of the hermaphrodite nervous system was accompanied by a decrease in wavelength of approximately equal magnitude (Fig. $5 A$ ). Similarly, feminization or masculinization of the male nervous system brought about changes in frequency that were accompanied by wavelength changes of equal proportion, although wavelength changes in males did not reach statistical significance (Fig. 5A). Because wave propagation speed is simply the product of frequency and wavelength, these observations imply that neural sex-reversal alters the frequency of body waves but not the speed of their propagation. Indeed, we found that the speed of wave propagation in neural sex-reversed animals was nearly indistinguishable from that of nontransgenic controls (Fig. 5E). This indicates that though sex-specific neural modifications are sufficient to regulate locomotor frequency, modifications to other aspects of the motor system may be required to fully implement sex-specific locomotor dynamics.

Interestingly, we found that animals with sex-reversed muscle exhibited wavelength changes that were largely independent of changes in locomotor frequency. Hermaphrodites with masculinized body wall muscle had increased wavelength relative to control animals, despite a modest increase in mean locomotor frequency (Fig. 5B). These changes led to a significant increase in the speed of wave propagation in muscle-masculinized hermaphrodites (Fig. 5E). Further, males with feminized muscle exhibited decreased wavelength, despite the absence of any detectable change in locomotor frequency (Fig. $5 B$ ). In contrast to musclemasculinized hermaphrodites, however, these animals showed no change in their wave speed (Fig. 5E). Thus, sexual modification of muscle can alter the speed of body wave propagation, such that masculinization of hermaphrodite muscle increases wave speed. These results are consistent with our previous analysis, which showed that when animals were swimming in saline solution and constrained only by their own neuromuscular and mechanical delays, the speed of male body waves was considerably 
A

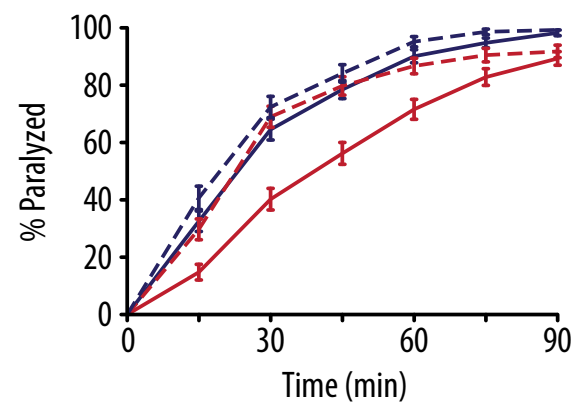

B Muscle feminization

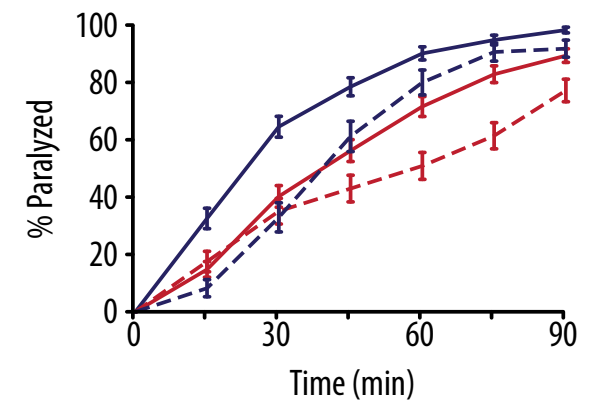

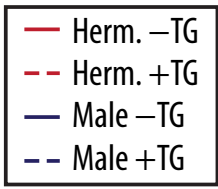

Figure 6. Sexual modification of muscle may regulate its sensitivity to cholinergic signals. $\boldsymbol{A}$, Compared to hermaphrodites, males exhibit greater sensitivity to the nematode-specific acetylcholine receptor agonist levamisole, as measured by the percentage of paralyzed worms in an exposed group over time. Masculinization of body wall muscle in hermaphrodites significantly increases their sensitivity to this agent. For the four experimental groups: - TG hermaphrodites, + TG hermaphrodites, $-\mathrm{TG}$ males, and + TG males, $n=169,158,172$, and 145, respectively. $\boldsymbol{B}$, Feminization of body wall muscle significantly decreases the sensitivity of males to the paralyzing effects of levamisole. For the four experimental groups: $-\mathrm{TG}$ hermaphrodites, + TG hermaphrodites, - TG males, and + TG males, $n=169,114,172$, and 145, respectively. Survival curves were generated using the Kaplan-Meier method and compared with the log-rank (Mantel-Cox) test.

faster than the speed of body waves in the hermaphrodite (Fig. $3 A$ ). A portion of this sex difference in wave speed might therefore be attributable to sex-specific properties of muscle.

Because sex-reversal of neurons and of body wall muscle altered wavelength in opposite directions, we considered the possibility that relieving muscular limitations on wave propagation speed might be important to facilitate male-typical highfrequency locomotion. We tested this by assessing the effects of simultaneous neural and muscle sex-reversal. Hermaphrodites carrying both neural and muscle masculinization transgenes exhibited an increase in locomotor frequency similar to that of animals carrying the neural masculinization transgene alone (Fig. $5 C$ ). However, in contrast to nervous system masculinization alone, hermaphrodites with both masculinized neurons and body wall muscle exhibited locomotor wavelength that was indistinguishable from that of control hermaphrodites (Fig. 5C). Similarly, simultaneous feminization of male neurons and muscle brought about locomotor frequency changes characteristic of neural sex-reversal without corresponding changes in wavelength (Fig. 5C). These observations support the idea that the sex of body wall muscle can indeed constrain the propagation of body waves under different locomotor dynamics. Moreover, sexreversal of neurons and muscle together restores the wild-type wavelength, suggesting that muscular and neural dynamics are tuned together to ensure a specific waveform geometry. An interesting implication of this observation is that the sex-specific shapes of body waves are ultimately determined by aspects of the body that are not affected by our sex-reversal transgenes.

To better understand how genetic sex might modify muscle properties, we compared the sensitivity of males and hermaphrodites to the cholinergic agonist levamisole. After incubation in levamisole-containing solutions, wild-type worms become paralyzed as a result of muscle hypercontraction (Rand, 2007). We found that males were significantly more susceptible to levamisole-mediated paralysis than hermaphrodites (Fig. 6), consistent with the possibility that male body-wall muscle responds more strongly or is more sensitive to cholinergic stimulation. Moreover, muscle-specific masculinization was sufficient to increase hermaphrodite levamisole sensitivity, and musclespecific feminization reduced male levamisole sensitivity (Fig. 6). Thus, at least some sex differences in muscle physiology are likely to be a direct consequence of the genetic sex of the muscle itself.

\section{Discussion}

In this study, we sought to use the simplicity and genetic tractability of the $C$. elegans model to clarify how a shared motor behavior is shaped by sexual modification of the motor system. In particular, we aimed to determine whether sexual modification of the nervous system played a significant role in this process. The results presented here speak to four major findings with respect to these goals. First, the kinematics of locomotion in C. elegans and related free-living nematodes are specialized according to sex in both their dynamics and geometry. These specializations are such that male locomotion is generally faster than that of the hermaphrodite or female. Second, kinematic sex differences are influenced by both the intrinsic dynamics of the motor system and by body-environment interactions. Modeling results suggest that either of these factors can contribute to sex differences in locomotor wavelength, with the influence of body-environment interaction becoming dominant at high levels of external load. Third, sex differences in locomotor frequency are determined primarily by sex-specific modification of shared neural circuitry. In particular, the sexual modification of shared sensory neurons is the predominant driver of sex-specific frequency. Fourth, sexspecific modification of body wall muscle acts together with neural modifications to fully implement sex-typical locomotor dynamics. That masculinization of body wall muscle is sufficient to elevate wave propagation speed in hermaphrodites suggests sex-specific muscle properties may constrain wave speed. Together, these findings demonstrate that the sexual differentiation of shared motor behavior entails more than simple reflex-like reactions to altered body mechanics. Rather, the genetic sexdetermination program implements a complex and coordinated set of modifications across multiple levels of the motor system, including shared neurons and musculature, to achieve optimal performance in distinct biomechanical contexts.

Perhaps one of the most striking findings of this study is that locomotor frequency is regulated by the sex-specific modification of shared neural circuitry. In particular, the sexual state of shared sensory neurons influences locomotor frequency so strongly that sex-reversal of these cells alone leads to nearly opposite-sex behavior on this measure (Fig. 4D). The notion that sensory neurons may regulate locomotor frequency has precedent in C. elegans, as dopaminergic and serotonergic sensory neurons are known to modulate frequency in response to food- 
related cues (Sawin et al., 2000). Sex-reversal of these sensory neuron subtypes, however, failed to influence locomotor frequency (Fig. 4C). These results must be interpreted with caution, as in most cases it is difficult to assess the completeness of sexreversal independent of locomotion. Nonetheless, these observations indicate that the focus of sexual modification may reside in a specific cell type not yet implicated in the regulation of locomotor kinematics. Identification of the cell types that are modified and their interactions with known components of the motor circuit has the potential to provide new insight into the modulation of pattern-generating circuits, and perhaps into the mechanisms of motor pattern generation itself.

Our results also implicate sexual modification of muscle function in the regulation of locomotor behavior. Interestingly, the effects of muscle sex-reversal seem to be complementary to those of neural sex-reversal. While pan-neural sex-reversal gives rise to opposite-sex locomotor frequency, it fails to sex-reverse the speed of wave propagation. This results in an altered locomotor wavelength: elevated locomotor frequency contracts the wavelength and decreased frequency expands it. Sex-reversal of body wall muscle has complementary effects on wavelength, with limited effects on locomotor frequency. In the case of hermaphrodites, this manipulation is sufficient to sex-reverse wave propagation speed. When neural sex-reversal is paired with muscular sex-reversal, locomotor frequency and wave speed are changed simultaneously, suppressing the wavelength abnormalities that arise when either manipulation is performed in isolation. Thus, it appears that neural and muscular dynamics are modulated in tandem to specify the wild-type waveform geometry. The question remains, however, as to how sex-specific muscle properties might act to constrain wave propagation. One possibility for this constraint is the well-known force-velocity tradeoff related to the length of muscle sarcomeres, whereby the contraction speed of muscle can be increased at the expense of its force-generating capacity (Alexander, 2003). It may be that the more flexible body of the male allows its muscle to operate in a high-frequency, low-force regime. Our levamisole results further raise the possibility that neuromuscular communication is also altered to give rise to changes in neuromuscular dynamics. Future work will show how sex-specific muscle dynamics are implemented at the molecular and cell-biological levels.

In these studies we used the biomechanical and proprioceptive control models developed by Fang-Yen et al. (2010) and Wen et al. (2012) to better understand the factors that lead to different locomotor kinematics in the sexes. Our investigations of swimming behavior indicate that sex differences in locomotor wavelength depend on internal neuromuscular delays when external loads are low, and on the ratio of bending stiffness to external drag when external loads are high (Fig. 3). Our sex-reversal data suggest sex differences in muscle dynamics may limit wave propagation speed under low mechanical load. At higher mechanical loads, the drag coefficient $C_{N}$ increases to where it dominates the expression determining wavelength. Males retain a longer wavelength under these circumstances due to their more slender body morphology, which exaggerates the phase difference between the waves of muscle torque and curvature traveling along the body (Fang-Yen et al., 2010). While important insights were gained from the use of models, it is also notable that model predictions consistently underestimated wavelengths observed in our data. One potential explanation for this discrepancy is that the values of certain model parameters were misestimated. Model parameters not directly measured in this study included bending stiffness and spatial extent of proprioceptive coupling in male animals, as well as the viscosity of the medium. While in all cases reasonable extrapolations of these values could be made based on data collected by others, future work-in particular, the direct measurement of male bending stiffness-should help mitigate these potential sources of error.

In our characterization of wild-type male nematode behavior, we observed that a more slender, agile adult male is characteristic of many C. elegans wild isolates, as well as other species of freeliving nematodes. The adaptive significance of these correlated sex differences in morphology and behavior may ultimately be related to the different reproductive strategies of the sexes. A direct correlation between body size and fecundity has been observed across a variety of invertebrate species (Hedrick and Temeles, 1989; Reeve and Fairbairn, 1999), including nematodes (Poulin, 1997). As our data suggest, a more slender body morphology may be important for efficient high-speed locomotion. Thus, hermaphrodites may face a trade-off between locomotor speed and fecundity. Larger, slower hermaphrodites may ultimately be more successful, as the reproductive fitness of hermaphrodites is critically dependent on the number of healthy embryos they can produce. Escaping this trade-off by simply lengthening the body may not be a viable option, as locomotion with multiple waves along the body can incur a penalty of increased drag and decreased locomotor efficiency (Dresdner et al., 1980). By contrast, male reproductive fitness is critically dependent upon the number of hermaphrodites that can be successfully fertilized. Free from the constraints of egg production, males may thus optimize their fitness by adopting a smaller, more slender body morphology that permits efficient high-frequency locomotion. Similar phenomena have been observed in other species with female-biased body-size dimorphism, where decreased body size enables males to increase their locomotor speed and compete more effectively for mates in a scramble competition scenario (Kelly et al., 2008). For nematodes, there may also be additional pressures for males to adopt a more slender body morphology, as deep body flexures are characteristic of male copulatory behavior in many nematode species (Fitch, 2000).

The robustness and adaptability of biological systems is of fundamental interest to a broad array of disciplines, and the distributed, decentralized nature of motor control systems is of particular interest in this regard. The adaptive modification of motor behaviors across the sexes of a species presents an important opportunity to understand how the properties of such systems are altered in a naturally occurring context. Our finding that sex differences in the locomotor behavior of $C$. elegans emerge from coordinated neural, muscular, and mechanical modifications reinforces the notion that distributed modification of the motor system may be necessary to achieve optimal behavior (Cohen, 1992). The motor behavior of C. elegans offers an outstanding model in which to gain new insight into how such distributed control systems are functionally modified.

\section{References}

Alexander RM (2003) Principles of animal locomotion. Princeton: Princeton UP.

Anderson DJ (2012) Optogenetics, sex, and violence in the brain: implications for psychiatry. Biol Psychiatry 71:1081-1089. CrossRef Medline

Ardizzi JP, Epstein HF (1987) Immunochemical localization of myosin heavy chain isoforms and paramyosin in developmentally and structurally diverse muscle cell types of the nematode Caenorhabditis elegans. J Cell Biol 105:2763-2770. CrossRef Medline

Barr MM, Garcia LR (2006) Male mating behavior. In: WormBook (Jorgensen EM, Kaplan JM, eds). http://www.wormbook.org. CrossRef

Berri S, Boyle JH, Tassieri M, Hope IA, Cohen N (2009) Forward locomo- 
tion of the nematode C. elegans is achieved through modulation of a single gait. HFSP J 3:186-193. CrossRef Medline

Brockie PJ, Madsen DM, Zheng Y, Mellem J, Maricq AV (2001) Differential expression of glutamate receptor subunits in the nervous system of Caenorhabditis elegans and their regulation by the homeodomain protein UNC-42. J Neurosci 21:1510-1522. Medline

Chalfie M, Sulston J, White J, Southgate E, Thomson J, Brenner S (1985) The neural circuit for touch sensitivity in Caenorhabditis elegans. J Neurosci 5:956-964.

Chiel HJ, Beer RD (1997) The brain has a body: adaptive behavior emerges from interactions of nervous system, body and environment. Trends Neurosci 20:553-557. CrossRef Medline

Clyne JD, Miesenböck G (2008) Sex-specific control and tuning of the pattern generator for courtship song in Drosophila. Cell 133:354-363. CrossRef Medline

Cohen AH (1992) The role of heterarchical control in the evolution of central pattern generators. Brain Behav Evol 40:112-124. CrossRef Medline

Colbert HA, Smith TL, Bargmann CI (1997) OSM-9, a novel protein with structural similarity to channels, is required for olfaction, mechanosensation, and olfactory adaptation in Caenorhabditis elegans. J Neurosci 17:8259-8269. Medline

Cronin CJ, Mendel JE, Mukhtar S, Kim YM, Stirbl RC, Bruck J, Sternberg PW (2005) An automated system for measuring parameters of nematode sinusoidal movement. BMC Genet 6:5. CrossRef Medline

Desai T, Batani D, Bernardinello A, Poletti G, Orsini F, Ullschmied J, Juha L, Skala J, Kralikova B, Krousky E, Pfeifer M, Kadlec C, Mocek T, Prag A, Renner O, Cotelli F, Lamia CL, Zullini A (2003) X-ray microscopy of living multicellular organisms with the Prague Asterix Iodine Laser System. Laser Part Beams 21:511-516.

de Vries GJ, Södersten P (2009) Sex differences in the brain: the relation between structure and function. Horm Behav 55:589-596. CrossRef Medline

Dresdner RD, Katz DF, Berger SA (1980) The propulsion by largeamplitude waves of uniflagellar microorganisms of finite length. J Fluid Mech 97:591-621. CrossRef

Eastman C, Horvitz HR, Jin Y (1999) Coordinated transcriptional regulation of the unc-25 glutamic acid decarboxylase and the unc-47 GABA vesicular transporter by the Caenorhabditis elegans UNC-30 homeodomain protein. J Neurosci 19:6225-6234. Medline

Emmons SW (2005) Male development. In: WormBook (Meyer BJ, ed). http://www.wormbook.org. CrossRef

Emmons SW (2006) Sexual behavior of the Caenorhabditis elegans male. Int Rev Neurobiol 69:99-123. Medline

Fang-Yen C, Wyart M, Xie J, Kawai R, Kodger T, Chen S, Wen Q, Samuel AD (2010) Biomechanical analysis of gait adaptation in the nematode Caenorhabditis elegans. Proc Natl Acad Sci U S A 107:20323-20328. CrossRef Medline

Field EF, Whishaw IQ (2008) Sex differences in the organization of movement. In: Sex differences in the brain: from genes to behavior (Becker JB, Berkley KJ, Geary N, Hampson E, Herman JP, eds), pp 155-175. New York: Oxford UP.

Fitch DH (2000) Evolution of "rhabditidae" and the male tail. J Nematol 32:235-244. Medline

Gray J, Hancock GJ (1955) The propulsion of sea-urchin spermatozoa. J Exp Biol 32:802-814.

Gray J, Lissmann HW (1964) The locomotion of nematodes. J Exp Biol 41:135-154. Medline

Haycraft CJ, Swoboda P, Taulman PD, Thomas JH, Yoder BK (2001) The C. elegans homolog of the murine cystic kidney disease gene Tg737 functions in a ciliogenic pathway and is disrupted in osm-5 mutant worms. Development 128:1493-1505. Medline

Hedrick AV, Temeles EJ (1989) The evolution of sexual dimorphism in animals: hypotheses and tests. Trends Ecol Evol 4:136-138. CrossRef Medline

Hodgkin J (1987) A genetic analysis of the sex-determining gene, tra-1, in the nematode Caenorhabditis elegans. Genes Dev 1:731-745. CrossRef Medline

Hodgkin JA (1974) Genetic and anatomical aspects of the Caenorhabditis elegans male. $\mathrm{PhD}$ thesis, University of Cambridge.

Hunter CP, Wood WB (1990) The tra-1 gene determines sexual phenotype cell-autonomously in C. elegans. Cell 63:1193-1204. CrossRef Medline

Kelly CD, Bussière LF, Gwynne DT (2008) Sexual selection for male mobil- ity in a giant insect with female-biased size dimorphism. Am Nat 172:417423. CrossRef Medline

Komatsu H, Mori I, Rhee JS, Akaike N, Ohshima Y (1996) Mutations in a cyclic nucleotide-gated channel lead to abnormal thermosensation and chemosensation in C. elegans. Neuron 17:707-718. CrossRef Medline

Lee K, Portman DS (2007) Neural sex modifies the function of a C. elegans sensory circuit. Curr Biol 17:1858-1863. CrossRef Medline

Li W, Feng Z, Sternberg P, Xu X (2006) A C. elegans stretch receptor neuron revealed by a mechanosensitive TRP channel homologue. Nature 440: 684-687. CrossRef Medline

Lighthill J (1976) Flagellar hydrodynamics. SIAM Rev 18:161-230. CrossRef

Lipton J, Kleemann G, Ghosh R, Lints R, Emmons SW (2004) Mate searching in Caenorhabditis elegans: a genetic model for sex drive in a simple invertebrate. J Neurosci 24:7427-7434. CrossRef Medline

Loer CM, Kenyon CJ (1993) Serotonin-deficient mutants and male mating behavior in the nematode Caenorhabditis elegans. J Neurosci 13:54075417. Medline

Lum DH, Kuwabara PE, Zarkower D, Spence AM (2000) Direct proteinprotein interaction between the intracellular domain of TRA-2 and the transcription factor TRA-1A modulates feminizing activity in C. elegans. Genes Dev 14:3153-3165. Medline

McGrath PT, Rockman MV, Zimmer M, Jang H, Macosko EZ, Kruglyak L, Bargmann CI (2009) Quantitative mapping of a digenic behavioral trait implicates globin variation in C. elegans sensory behaviors. Neuron 61: 692-699. CrossRef Medline

Mehra A, Gaudet J, Heck L, Kuwabara PE, Spence AM (1999) Negative regulation of male development in Caenorhabditis elegans by a proteinprotein interaction between TRA-2A and FEM-3. Genes Dev 13:14531463. CrossRef Medline

Mello CC, Fire A (1995) DNA transformation. In: Caenorhabditis elegans: modern biological analysis of an organism (Epstein HF, Shakes DC, eds). San Diego: Academic.

Mowrey WR, Portman DS (2012) Sex differences in behavioral decisionmaking and the modulation of shared neural circuits. Biol Sex Differ 3:8. CrossRef Medline

Nass R, Hall DH, Miller DM 3rd, Blakely RD (2002) Neurotoxin-induced degeneration of dopamine neurons in Caenorhabditis elegans. Proc Natl Acad Sci U S A 99:3264-3269. CrossRef Medline

Nishikawa K, Biewener AA, Aerts P, Ahn AN, Chiel HJ, Daley MA, Daniel TL, Full RJ, Hale ME, Hedrick TL, Lappin AK, Nichols TR, Quinn RD, Satterlie RA, Szymik B (2007) Neuromechanics: an integrative approach for understanding motor control. Integr Comp Biol 47:16-54. CrossRef Medline

Nonet ML, Staunton JE, Kilgard MP, Fergestad T, Hartwieg E, Horvitz HR, Jorgensen EM, Meyer BJ (1997) Caenorhabditis elegans rab-3 mutant synapses exhibit impaired function and are partially depleted of vesicles. J Neurosci 17:8061-8073. Medline

Park SJ, Goodman MB, Pruitt BL (2007) Analysis of nematode mechanics by piezoresistive displacement clamp. Proc Natl Acad Sci U S A 104: 17376-17381. CrossRef Medline

Perkins LA, Hedgecock EM, Thomson JN, Culotti JG (1986) Mutant sensory cilia in the nematode Caenorhabditis elegans. Dev Biol 117:456-487. CrossRef Medline

Portman DS (2007) Genetic control of sex differences in C. elegans neurobiology and behavior. Adv Genet 59:1-37. CrossRef Medline

Poulin R (1997) Covariation of sexual size dimorphism and adult sex ratio in parasitic nematodes. Biol J Linn Soc 62:567-580. CrossRef

Rand JB (2007) Acetylcholine. In: WormBook (Joregensen EM, Kaplan JM, eds) http://www.wormbook.org. CrossRef

Reeve JP, Fairbairn DJ (1999) Change in sexual size dimorphism as a correlated response to selection on fecundity. Heredity 83:697-706. CrossRef Medline

Sawin ER, Ranganathan R, Horvitz HR (2000) C. elegans locomotory rate is modulated by the environment through a dopaminergic pathway and by experience through a serotonergic pathway. Neuron 26:619-631. CrossRef Medline

Schafer WF (2006) Genetics of egg-laying in worms. Annu Rev Genet 40: 487-509. CrossRef Medline

Schindelman G, Whittaker AJ, Thum JY, Gharib S, Sternberg PW (2006) 
Initiation of male sperm-transfer behavior in Caenorhabditis elegans requires input from the ventral nerve cord. BMC Biol 4:26. CrossRef Medline

Sternberg P (2005) Vulval development. In: WormBook (Meyer BJ, ed) http://www.wormbook.org. CrossRef

Sulston JE, Horvitz HR (1977) Postembryonic cell lineages of the nematode Caenorhabditis elegans. Dev Biol 56:110-156. CrossRef Medline

Sze JY, Victor M, Loer C, Shi Y, Ruvkun G (2000) Food and metabolic signalling defects in a Caenorhabditis elegans serotonin-synthesis mutant. Nature 403:560-564. CrossRef Medline

Tirtaatmadja V, Dunstan DE, Boger DV (2001) Rheology of dextran solutions. J Non-Newtonian Fluid Mech 97:295-301. CrossRef

Troemel ER, Chou JH, Dwyer ND, Colbert HA, Bargmann CI (1995) Divergent seven transmembrane receptors are candidate chemosensory receptors in C. elegans. Cell 83:207-218. CrossRef Medline

Weber KP, De S, Kozarewa I, Turner DJ, Babu MM, de Bono M (2010) Whole genome sequencing highlights genetic changes associated with laboratory domestication of C. elegans. PLOS One 5:e13922. CrossRef Medline
Wen Q, Po MD, Hulme E, Chen S, Liu X, Kwok SW, Gershow M, Leifer AM, Butler V, Fang-Yen C, Kawano T, Schafer WR, Whitesides G, Wyart M, Chklovskii DB, Zhen M, Samuel AD (2012) Proprioceptive Coupling within Motor Neurons Drives C. elegans Forward Locomotion. Neuron 76:750-761. CrossRef Medline

White JG (1988) The anatomy. In: The nematode Caenorhabditis elegans (Wood WB, ed). Woodbury, NY: Cold Spring Harbor Laboratory.

White JG, Southgate E, Thomson JN, Brenner S (1986) The structure of the nervous system of the nematode Caenorhabditis elegans. Philos Trans R Soc Lond B 314:1-340. CrossRef

White JQ, Nicholas TJ, Gritton J, Truong L, Davidson ER, Jorgensen EM (2007) The sensory circuitry for sexual attraction in C. elegans males. Curr Biol 17:1847-1857. CrossRef Medline

Winnier AR, Meir JY, Ross JM, Tavernarakis N, Driscoll M, Ishihara T, Katsura I, Miller DM 3rd (1999) UNC-4/UNC-37-dependent repression of motor neuron-specific genes controls synaptic choice in Caenorhabditis elegans. Genes Dev 13:2774-2786. CrossRef Medline

Wolff JR, Zarkower D (2008) Somatic sexual differentiation in Caenorhabditis elegans. Curr Top Dev Biol 83:1-39. CrossRef Medline 